\title{
Experiência de gravidez e aborto em crianças, adolescentes e jovens em situação de rua
}

\author{
Pregnancy and abortion experience among children, adolescents \\ and youths living on the streets
}

\author{
Lucas Neiva-Silva ${ }^{1}$ \\ Lauro Miranda Demenech ${ }^{1}$ \\ Laísa Rodrigues Moreira ${ }^{1}$ \\ Adriano Trassantes Oliveira ${ }^{1}$ \\ Fernanda Torres de Carvalho ${ }^{2}$ \\ Simone dos Santos Paludo ${ }^{1}$
}

${ }^{1}$ Programa de PósGraduação em Saúde Pública, Universidade Federal do Rio Grande (FURG). Av. Itália km 8/ Campus Carreiros/Ed. Psicologia 35, Carreiros. 96201-900 Rio Grande RS Brasil. lucasneivasilva@gmail.com ${ }^{2}$ Coordenação Estadual de IST/Aids, Secretaria Estadual de Saúde do RS. Porto Alegre RS Brasil.
Abstract The aim of this study is to identify the prevalence of pregnancy and abortion experience, along with associated factors among street children, adolescents and youths in Porto Alegre and Rio Grande, RS, Brazil. The sampling method in this cross-sectional study was Respondent-Driven Sampling (RDS), innovative in research about hidden populations. Interviews were conducted with 307 subjects, aged 10 to 21 years. In multivariate analysis, Poisson regression with a robust error variance was used. Most of this sample were males, with no contact with school and family. Almost half of the participants had been on the street for five years or more, spending more than 15 hours per day on the streets. The prevalence of pregnancy experience was 29.3\%, independently associated with being a girl, having 10 sexual partners or more in the last year, having a fixed sexual partner in the last year, and being older. The prevalence of abortion experience was $10.4 \%$, independently associated with not living with the family, having more than two pregnancies, and being younger. The high prevalence of pregnancy and abortion experience highlights the need for the development of better sexual and reproductive health policies focused on this population.

Key words Pregnancy, Abortion, Street adolescents, Street youth, Teenage pregnancy
Resumo O objetivo deste estudo é identificar a prevalência de experiência de gravidez e aborto $e$ os fatores associados em crianças, adolescentes e jovens em situação de rua, das cidades de Porto Alegre e Rio Grande, RS, Brasil. Neste estudo transversal, como método de amostragem, foi utilizado o Respondent-Driven Sampling (RDS), inovador em pesquisas com populações de difícil acesso. Foram entrevistados 307 indivíduos, com idade entre 10 e 21 anos. Na análise multivariada foi utilizada regressão de Poisson, com ajuste robusto da variância. A maioria dos participantes foi do sexo masculino, sem vínculos com escola e com a família. Quase metade estava há cinco anos ou mais na rua, permanecendo mais de 15 horas diárias nela. A prevalência de experiência de gravidez foi de 29,3\%, estando independentemente associada com ser do sexo feminino, ter mais de 10 parceiros sexuais no último ano, ter parceiro sexual fixo no último ano e ter mais idade. A prevalência de experiência de aborto foi de 10,4\%, estando associado com não morar com a família, ter duas ou mais gravidezes e ter menos idade. A alta prevalência de experiência de gravidez e aborto aponta para a necessidade de melhores políticas de saúde sexual e reprodutiva específicas para esta população.

Palavras-chave Gravidez, Aborto, Adolescentes em situação de rua, Jovens em situação de rua, Gravidez na adolescência 


\section{Introdução}

Crianças e adolescentes em situação de rua estão mais propensas a comportamentos de risco e mais vulneráveis a uma série de desfechos negativos para a saúde quando comparadas a crianças e adolescentes da população geral ${ }^{1-3}$. Entre os comportamentos de risco estão o início precoce da vida sexual e a prática de sexo inseguro, aumentando a probabilidade de gravidez indesejada e de aborto nessa população ${ }^{4-6}$.

De todos os partos que ocorrem com pessoas abaixo de 18 anos no mundo, cerca de $95 \%$ ocorrem em países em desenvolvimento. Nestes países, $19 \%$ de todas as gravidezes ocorrem nesta faixa etária ${ }^{7}$. Pesquisas sobre gravidez com a população de adolescentes em situação de rua são escassas. Contudo, os achados existentes revelam a necessidade de tratar esse tema de forma integral, considerando as implicações para a saúde e desenvolvimento. Nos EUA, um estudo conduzido com adolescentes entre 14 e 17 anos, encontrou prevalência de gravidez de $48 \%$ entre os que passavam as noites nas ruas, $33 \%$ nos que moravam em abrigos e menos de $10 \%$ nos adolescentes que residiam com suas famílias ${ }^{3}$. Em outro estudo estadunidense, foi verificado que $53 \%$ das adolescentes em situação de rua, com idades entre 15 e 22 anos, engravidaram pelo menos uma vez na vida ${ }^{8}$. Nessa mesma direção, uma pesquisa realizada em Montreal, no Canadá, revelou que $41,8 \%$ das adolescentes em situação de rua de 14 a 19 anos já havia engravidado. Pesquisa realizada em três cidades da Ucrânia encontrou que $41,7 \%$ das meninas e $23,5 \%$ dos meninos em situação de rua, com idades entre 15 e 24 anos, já tiveram experiência de gravidez ${ }^{10}$.

Uma das possíveis consequências da gestação indesejada na adolescência é o aborto. Na população de adolescentes em situação de rua, estudos realizados nos EUA e na Ucrânia estimam que a prevalência de experiência de aborto, dentre os que tiveram experiência de gravidez, seja de aproximadamente $43 \%{ }^{8,10}$. Quando observada a população mundial, estima-se que $14 \%$ de todos os abortos acontecem em mulheres com menos de 20 de idade ${ }^{11}$.

No Brasil, 2,9\% das adolescentes da população geral, na faixa etária de 10 a 17 anos, já tiveram pelo menos um filho ${ }^{12}$. Um estudo realizado com 6935 adolescentes, de ambos os sexos, entre 14 e 19 anos, das cinco regiões brasileiras, mostrou que $3,3 \%$ relataram ter experiência de gravidez e 1,3\% relataram experiência de aborto ${ }^{13}$. Já na população em situação de rua, essa realidade se mostra diferente. Em um dos poucos estudos brasileiros sobre o tema, identificou-se que $23,2 \%$ dos meninos em situação de rua e 51,9\% das meninas, entre 9 e 18 anos, tiveram experiência de gravidez ${ }^{14}$. Neste estudo, $26,4 \%$ do total de meninas sexualmente ativas já haviam abortado. A Pesquisa Nacional de Aborto (PNA) encontrou que dentre as mulheres que já abortaram, 17\% realizaram o último aborto quando tinham entre 12 e 17 anos $^{15}$.

Entre os principais fatores associados à gravidez e ao aborto em jovens em situação de rua estão: estar em situação de rua desde muito cedo ${ }^{6}$, estar fora de casa por longos períodos de tempo e desistência da escola ${ }^{16}$, início precoce da vida sexual ${ }^{10}$ e praticar sexo em troca de dinheiro, favores ou vantagens ${ }^{5}$.

A população em situação de rua é diversificada e flutuante, variando de acordo com o total de horas passadas na rua e com alta rotatividade do local de permanência ${ }^{17}$. Além disto, o uso de distintas definições desta população entre os pesquisadores dificulta ainda mais a comparabilidade dos resultados encontrados ${ }^{5,18}$. Em virtude disso, torna-se complexo quantificar o total de indivíduos em situação de rua. Entretanto, no Brasil, um levantamento nacional realizado nas 27 capitais encontrou 2807 crianças e adolescentes em situação de rua ${ }^{17}$.

A realidade da rua revela que ainda são necessárias pesquisas com técnicas de amostragem que levem em consideração a natureza complexa e mutável desta população-alvo. Associado a isso, existe uma lacuna no conhecimento sobre a experiência de gravidez e aborto em crianças e adolescentes em situação de rua brasileiros. A partir do exposto, o objetivo deste estudo é identificar a prevalência de experiência de gravidez e de aborto e os fatores associados em crianças, adolescentes e jovens em situação de rua, com idades entre 10 e 21 anos, nas cidades de Porto Alegre e Rio Grande (RS, Brasil).

\section{Método}

\section{Delineamento e participantes}

Trata-se de estudo transversal de caráter analítico, cujos participantes foram crianças, adolescentes e jovens em situação de rua, com idades entre 10 e 21 anos, nas cidades de Porto Alegre e Rio Grande (RS, Brasil), no ano de 2008. Para o Estatuto da Criança e do Adolescente, são considerados crianças os indivíduos de até 12 anos de 
idade incompletos, e adolescentes aqueles entre 12 e 18 anos de idade ${ }^{19}$. Estar em situação de rua foi caracterizado como: passar parte do tempo diário no espaço da rua; realizar atividades no contexto da rua, incluindo trabalhar informalmente, brincar e perambular; e estar desacompanhado de um adulto responsável ${ }^{17,18,20}$. Este estudo é parte de uma pesquisa maior que investigou as condições de saúde e vulnerabilidade de crianças, adolescentes e jovens em situação de rua em Porto Alegre e Rio Grande ${ }^{21}$. Porto Alegre é a capital do estado do Rio Grande do Sul e conta com aproximadamente 1.410 .000 habitantes. Rio Grande é um município portuário do mesmo estado, com uma população de 197 mil habitantes ${ }^{12}$.

\section{Amostragem e cálculo amostral}

O método de amostragem utilizado foi o Respondent-Driven Sampling (RDS) ou Amostragem Conduzida pelo Participante. O RDS combina características da amostragem pelo método "bola de neve", com modelos matemáticos que "pesam" a amostra para compensar o fato de que esta foi coletada, inicialmente, de uma maneira não-randômica. Este método utiliza tecnologias, a partir dos princípios da teoria de Markov, em que longas cadeias de referência de indivíduos produzem uma amostra final independente daqueles que a iniciaram ${ }^{22}$.

O RDS pressupõe que as melhores pessoas para recrutarem membros de populações de difícil acesso são seus próprios pares ${ }^{22}$. As chamadas "populações de difícil acesso" (hard to reach populations) apresentariam duas características principais: 1) não existe uma estrutura amostral definida, em que tanto o número de indivíduos quanto as fronteiras divisórias da população são desconhecidos; e 2) existe uma forte preocupação em relação à privacidade, principalmente porque os membros destas populações geralmente são estigmatizados ou estão associados a comportamentos ilícitos, o que leva os indivíduos a se recusarem a participar das pesquisas ou a fornecer respostas não-confiáveis. Esta técnica vem sendo cada vez mais difundida e utilizada em estudos recentes, confirmando a viabilidade do método para acessar tais populações de forma rápida e eficiente ${ }^{23,24}$.

Quando métodos de amostragem em cadeia - como o bola de neve - são modelados estatisticamente, é possível derivar indicadores estatisticamente válidos e também determinar quantitativamente a sua precisão ${ }^{25}$. Assim como em outros métodos de amostragem probabilística, é possível avaliar a confiabilidade dos dados obtidos e torna possível a realização de inferências sobre as características da população a partir da qual a amostra foi obtida. Através do RDS, é possível iniciar a seleção da amostra por um processo não probabilístico, tendo a possibilidade de alcançar uma amostra final probabilística. Análises são realizadas, (por meio do software RDSAT, atualmente na versão 7.1$)^{26}$, que permitem avaliar a amostra final alcançada e definir se a mesma alcançou características de uma amostra probabilística ${ }^{27}$. Isto ocorre quando as características da amostra se tornam estáveis, alcançando o chamado "equilíbrio"25,27.

O processo amostral foi iniciado com a identificação de crianças, adolescentes e jovens, chamados de sementes. A seleção das sementes ocorreu de forma não-aleatória, na tentativa de representar a diversidade social e geográfica da população. Através de reuniões com equipes de organizações governamentais (OGs) e não-governamentais (ONGs) de ambas as cidades, foram escolhidas pessoas com uma intensa rede social, as quais ficaram responsáveis pelo recrutamento dos próximos participantes. A cada participante-semente foram entregues dois cupons-convite (numerados com código específico que possibilitou a organização sequencial de recrutamento), a serem distribuídos a outros dois conhecidos, que também estivessem em situação de rua, os quais compuseram a primeira onda. Cada um destes sujeitos, depois de serem entrevistados, receberam dois outros cupons, que foram entregues aos novos participantes da terceira onda; e assim sucessivamente.

Cada participante recebeu um ressarcimento, na forma de vale-alimentação, pela entrevista realizada e outro por cada participante recrutado de maneira efetiva, ou seja, após o convidado também ter sido entrevistado. O RDS pressupõe que o fato de ser a própria criança/adolescente quem convida o próximo participante tende a reduzir as "respostas socialmente aceitas", na medida em que é menor a desconfiança em relação a um convite feito pelo pesquisador, além de serem recrutados participantes aos quais os pesquisadores, de fato, não teriam acesso.

Em função do projeto maior investigar diferentes temáticas, foi realizado um cálculo amostral descritivo, usando como desfecho o uso de preservativos, com prevalência esperada de $26 \%$. Apesar de não existir um marco de amostragem definido, foi utilizado como referência um estudo realizado na cidade de Porto Alegre, com esta 
população, em que se estimou 450 adolescentes e jovens em situação de rua ${ }^{4}$. Os parâmetros utilizados foram intervalo de confiança de $95 \%$, com margem de erro de 6 pontos percentuais e poder de $80 \%$. Em função da técnica de amostragem, foi assumido um efeito de delineamento de 1,25. Ao total ainda foi acrescido $10 \%$, para compensar possíveis desistências. Com base nisso, estimouse uma amostra de 195 participantes em Porto Alegre. Como não havia estimativa da população de crianças e adolescentes em situação de rua em Rio Grande, foi adotado como critério o valor relativo a um terço da amostra de Porto Alegre, correspondendo a 65 indivíduos para Rio Grande. Assim, foram planejadas 5 sementes em Porto Alegre e 2 sementes em Rio Grande, estimandose cinco ondas por semente e $30 \%$ de interrupções na sequência de recrutamento. Devido a estas interrupções, ao longo da coleta de dados, foram inseridas duas novas sementes em Porto Alegre, perfazendo um total de 7 sementes nesta cidade.

\section{Instrumentos, variáveis e logística}

Foi realizada uma entrevista com cada participante a partir de um questionário fechado, construído com base em instrumentos utilizados em outros estudos com essa população $0^{4,18}$. Para este artigo, foram analisadas as seções do questionário referentes às variáveis: 1$)$ sociodemográficas - sexo (masculino/feminino), idade (em anos completos), vínculo com a escola (nunca estudou ou parou de estudar/estuda), morar com a família (não/sim), anos em situação de rua, horas na rua por dia; 2) sobre sexualidade: já teve relações sexuais (não/sim), idade da primeira relação sexual (em anos), número de parceiros sexuais no último ano, teve parceiro/a fixo/a no último ano (não/sim), carregar preservativo consigo no último mês (não/sim) e fez sexo em troca de dinheiro, favores ou vantagens (não/sim); 3) sobre gravidez: experiência de gravidez (não/sim), idade da primeira gravidez (em anos completos), número de vezes que esteve grávida/engravidou alguém, fez exame pré-natal durante a última gravidez (não/sim); 4) sobre aborto: experiência de aborto (não/sim), número de abortos, tipo de aborto na primeira vez que abortou (natural/ provocado). O termo "experiência de gravidez" é utilizado para explicitar que a variável é investigada tanto em pessoas do sexo feminino quanto do sexo masculino.
A equipe de entrevistadores foi treinada sobre métodos de abordagem da população-alvo, técnicas de entrevista e padronização de respostas. As entrevistas foram realizadas em locais abertos, como ruas, praças e parques. Os locais de entrevista foram definidos buscando contextos com menor chance de interrupção da entrevista, bem como observando o fator segurança para os participantes e entrevistadores.

\section{Análise de dados}

Os dados foram tabulados e migrados para o RDSAT 7.1. Análises das amostras indicaram que as mesmas atingiram o equilíbrio, tanto na cidade de Porto Alegre quanto em Rio Grande. Análises complementares foram realizadas utilizando os programas SPSS $17.0^{28}$ e STATA $13.1^{29}$.

Inicialmente foi realizada análise univariada para descrição da amostra, calculando a frequência das variáveis independentes e dos desfechos (experiência de gravidez e experiência de aborto). Em análise bivariada, foram calculadas as prevalências de ambos os desfechos para os grupos das variáveis independentes. Foram utilizados os testes de Wald para heterogeneidade e de tendência linear para testar a significância estatística das associações. As diferenças entre os grupos foram descritas em razões de prevalência, com os respectivos intervalos de confiança de 95\% e valores $p$.

Para a análise ajustada, foi utilizada a Regressão de Poisson com ajuste robusto da variância ${ }^{30}$. Para isto, foi elaborado um Modelo Hierárquico de Análise para cada desfecho (Figura 1). Para a seleção das variáveis para o modelo final, foi utilizado o método backward. Mantiveram-se no modelo apenas aquelas variáveis com valor $p \leq 0,2$, como estratégia de controle de possível confusão. Foram utilizados os mesmos testes descritos na análise bivariada.

\section{Aspectos éticos}

O projeto de pesquisa foi aprovado pelo Comitê de Ética em Pesquisa da FURG. A Vara da Infância e da Juventude das cidades de Porto Alegre e de Rio Grande concedeu permissão para entrevista com crianças e adolescentes. Houve Consentimento Livre e Esclarecido verbal para maiores de 18 anos que não sabiam ler e escrito para os que sabiam ler. $\mathrm{O}$ estudo maior contou com o financiamento do Programa Nacional de DST/HIV-AIDS do Ministério da Saúde. 


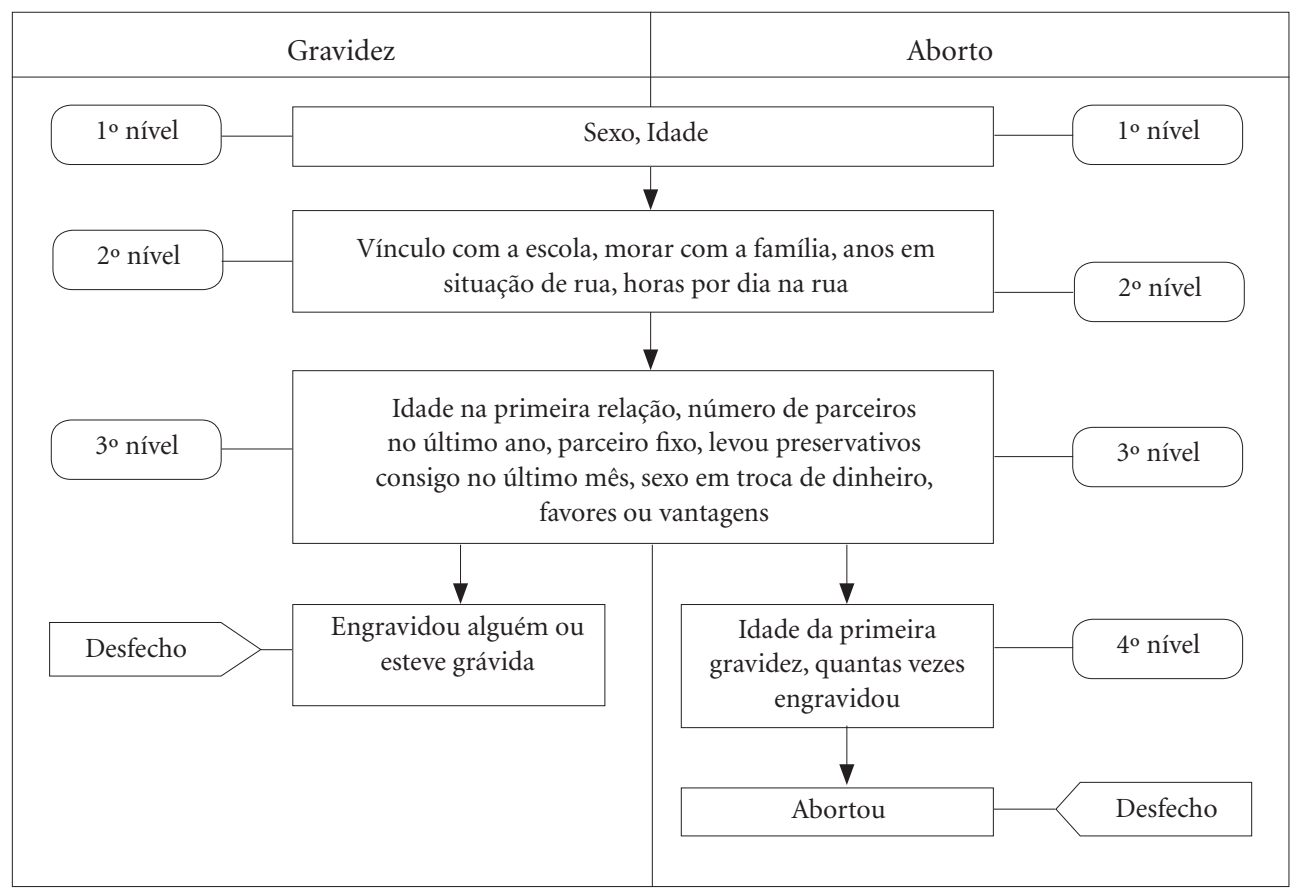

Figura 1. Modelo Hierárquico de Análise para cada desfecho.

\section{Resultados}

Participaram 307 crianças, adolescentes e jovens em situação de rua, sendo 204 entrevistados em Porto Alegre e 103 em Rio Grande. A amostra foi composta predominantemente por adolescentes e jovens $(93,8 \%)$, sendo a maior parte do sexo masculino (81,1\%). Dos entrevistados, 54,7\% nunca estudou ou parou de estudar e 52,1\% não morava com a família. Quase metade da amostra (45,6\%) estava há 5 anos ou mais em situação de rua, e 40,3\% permanecia mais de 15 horas diárias na rua. Da amostra total, 77,9\% tiveram relações sexuais alguma vez na vida, sendo que $21 \%$ iniciaram a vida sexual ainda na infância. Sexo em troca de dinheiro, favores ou vantagens ocorreu em 18,9\% (Tabela 1).

A experiência de gravidez (ter ficado grávida ou ter engravidado alguém) ocorreu em aproximadamente um terço da amostra $(29,3 \%)$. A prevalência de gravidez foi maior no grupo das meninas $(59,5 \%)$ do que nos meninos $(33,1 \%)$. Dos que já tiveram experiência de gravidez, 42,2\% a tiveram mais de uma vez, e 26,7\% engravidaram com idade igual ou inferior a 14 anos. Apenas $57,8 \%$ fez algum exame pré-natal durante a gravidez. Além disso, um terço daqueles que tiveram experiência de gravidez já abortaram, correspon-
Tabela 1. Descrição da amostra de crianças, adolescentes e jovens em situação de rua de acordo com as variáveis estudadas. Porto Alegre e Rio Grande/RS (N = 307).

\begin{tabular}{lrr}
\multicolumn{1}{c}{ Variável } & N & $\%$ \\
\hline Sexo $(\mathrm{n}=307)$ & 249 & 81,1 \\
$\quad$ Masculino & 58 & 18,9 \\
$\quad$ Feminino & & \\
Idade (anos) $(\mathrm{n}=307)$ & 19 & 6,2 \\
$\quad \leq 11$ & 78 & 25,4 \\
$12-14$ & 89 & 29,0 \\
$15-17$ & 121 & 39,4 \\
$18-21$ & & \\
Vínculo com a escola $(\mathrm{n}=307)$ & 168 & 54,7 \\
Nunca estudou ou parou de estudar & 139 & 45,3 \\
Estuda & & \\
Morar com a família $(\mathrm{n}=307)$ & 160 & 52,1 \\
Não & 147 & 47,9 \\
Sim & & \\
Anos em situação de rua $(\mathrm{n}=307)$ & 167 & 54,4 \\
$\leq 4$ & 92 & 30,0 \\
$5-9$ & 48 & 15,6 \\
$\geq 10$ & & \\
Horas na rua por dia $(\mathrm{n}=307)$ & 111 & 36,2 \\
$\leq 7$ & 72 & 23,5 \\
$8-14$ & 124 & 40,3 \\
$\geq 15$ & continua
\end{tabular}


Tabela 1. continuação

\begin{tabular}{|c|c|c|}
\hline Variável & $\mathbf{N}$ & $\%$ \\
\hline \multicolumn{3}{|c|}{ Já teve relações sexuais $(\mathrm{n}=307)$} \\
\hline Não & 68 & 22,1 \\
\hline Sim & 239 & 77,9 \\
\hline \multicolumn{3}{|c|}{$\begin{array}{l}\text { Idade da } 1^{\text {a }} \text { relação sexual (anos) } \\
(\mathrm{n}=239)\end{array}$} \\
\hline$\leq 11$ & 50 & 21,0 \\
\hline 12 a 14 & 121 & 50,9 \\
\hline 15 a 17 & 61 & 25,6 \\
\hline$\geq 18$ & 6 & 2,5 \\
\hline \multicolumn{3}{|c|}{$\begin{array}{l}\text { Número de parceiros sexuais no último } \\
\text { ano }(\mathrm{n}=237)\end{array}$} \\
\hline$\leq 10$ & 196 & 82,7 \\
\hline$>10$ & 41 & 17,3 \\
\hline \multicolumn{3}{|c|}{$\begin{array}{l}\text { Teve parceiro/a fixo/a no último ano } \\
(\mathrm{n}=238)\end{array}$} \\
\hline Não & 73 & 30,7 \\
\hline Sim & 165 & 69,3 \\
\hline \multicolumn{3}{|c|}{$\begin{array}{l}\text { Carregou preservativo consigo no } \\
\text { último mês }(\mathrm{n}=307)\end{array}$} \\
\hline Não & 152 & 49,5 \\
\hline Sim & 155 & 50,5 \\
\hline \multicolumn{3}{|c|}{$\begin{array}{l}\text { Fez sexo em troca de dinheiro, favores } \\
\text { ou vantagens }{ }^{*}(n=307)\end{array}$} \\
\hline Não & 249 & 80,1 \\
\hline Sim & 58 & 18,9 \\
\hline \multicolumn{3}{|c|}{ 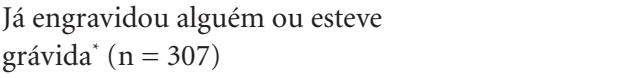 } \\
\hline Não & 217 & 70,7 \\
\hline Sim & 90 & 29,3 \\
\hline \multicolumn{3}{|c|}{ Idade da $1^{\text {a }}$ gravidez $($ anos $)(n=90)$} \\
\hline$\leq 14$ & 24 & 26,7 \\
\hline $15-17$ & 41 & 45,6 \\
\hline$\geq 18$ & 25 & 27,8 \\
\hline \multicolumn{3}{|c|}{ Número de gravidezes $(\mathrm{n}=90)$} \\
\hline 1 & 52 & 57,8 \\
\hline$\geq 2$ & 38 & 42,2 \\
\hline \multicolumn{3}{|c|}{$\begin{array}{l}\text { Fez exame pré-natal durante a última } \\
\text { gravidez }(\mathrm{n}=90)\end{array}$} \\
\hline Não & 15 & 16,7 \\
\hline Sim & 52 & 57,8 \\
\hline Não sabe & 23 & 25,5 \\
\hline \multicolumn{3}{|c|}{ Já fez algum aborto? $(\mathrm{n}=307)^{*}$} \\
\hline Não & 275 & 89,6 \\
\hline Sim & 32 & 10,4 \\
\hline \multicolumn{3}{|c|}{ Tipo de aborto na $1^{\text {a }}$ vez $(\mathrm{n}=32)$} \\
\hline Natural & 18 & 56,3 \\
\hline Provocado & 14 & 43,7 \\
\hline \multicolumn{3}{|l|}{ Número de abortos $(\mathrm{n}=32)$} \\
\hline 1 & 21 & 65,6 \\
\hline$\geq 2$ & 11 & 34,4 \\
\hline
\end{tabular}

N: frequência absoluta; \%: frequência relativa. " Percentual calculado sobre o número total de casos, agrupando "não" e "não se aplica" na mesma categoria. dendo a $10,4 \%$ da amostra total. Das crianças, adolescentes e jovens que abortaram, 34,4\% já abortaram mais de uma vez (Tabela 1). Estratificando por sexo, $17,4 \%$ das meninas entrevistadas relataram já ter abortado, enquanto que $8,8 \%$ dos meninos referiram a experiência de aborto.

$\mathrm{Na}$ análise bivariada, conforme a Tabela 2, foram identificados alguns fatores de risco para a gravidez. Levando em consideração a prevalência de gravidez de $29,3 \%$ e a razão expostos/não-expostos de 1 para 4, este estudo atingiu Poder de $84,0 \%$ para identificar associações com razões de prevalência de pelo menos 1,8 . Os fatores de risco foram ser do sexo feminino (RP: 1,79; IC95\%: 1,30-2,47), nunca estudar ou ter parado de estudar (RP: 3,57; IC95\%: 2,02-6,31), não morar com a família (RP: 2,18; IC95\%: 1,40-3,40), ter tido mais de 10 parceiros sexuais no último ano (RP: 1,63; IC95\%: 1,17-2,28), ter tido parceiro fixo no último ano (RP: 1,66; IC95\%: 1,09-2,65) e ter feito sexo em troca de dinheiro ou favores (RP: 1,88; IC95\%: 1,39-2,56). Da mesma forma, houve uma tendência a aumentar a probabilidade do participante ter a experiência de gravidez conforme tinha mais anos em situação de rua e horas diárias dispendidas na rua ( $p$ de tendência linear $<0,001$ para ambos).

Em relação ao aborto, ter duas ou mais gravidezes apareceu como fator de risco (RP: 3,01 IC95\%: 1,61-5,61) (Tabela 2). Além disto, houve uma diminuição na probabilidade de ter aborto conforme $\mathrm{o}$ aumento na idade do participante ( $\mathrm{p}$ de tendência linear $=0,007$ ), e um aumento na probabilidade de aborto quanto menor a idade do indivíduo na primeira gravidez ( $\mathrm{p}$ de tendência linear $<0,001$ ).

$\mathrm{Na}$ análise ajustada para experiência de gravidez (Tabela 3), mantiveram-se como fatores de risco ser do sexo feminino (RP: 1,70 IC95\%: $1,30-2,22)$, ter tido mais de 10 parceiros sexuais no último ano (RP: 1,41 IC95\%: 1,02-1,94) e ter tido parceiro fixo no último ano (RP: 1,66 IC95\%: 1,13-2,42). Além disto, houve um aumento na probabilidade de ter a experiência de gravidez conforme o aumento da idade ( $p$ de tendência linear $<0,001)$ e conforme o aumento no tempo em situação de rua ( $p$ de tendência linear $=0,006$ ). As variáveis independentes "morar com a família", "horas diárias na rua" e "sexo em troca de dinheiro ou favores" perderam o efeito na análise ajustada. Apesar da variável "nunca ter estudado ou parado de estudar" ter se mantido no modelo final da regressão para controle de confusão, esta não manteve a associação estatisticamente significativa. 
Tabela 2. Prevalência de experiência de gravidez e de aborto de acordo com as características de crianças, adolescentes e jovens em situação de rua das cidades de Porto Alegre e Rio Grande/RS. $(\mathrm{N}=307)$. Análise bivariada (bruta).

\begin{tabular}{|c|c|c|c|c|c|c|}
\hline \multirow{2}{*}{ Variável } & \multicolumn{3}{|c|}{ Experiência de Gravidez } & \multicolumn{3}{|c|}{ Experiência de Aborto } \\
\hline & $\mathrm{N}(\%)$ & RP (IC95\%) & $p^{*}$ & $\mathrm{~N}(\%)$ & RP (IC95\%) & $p^{*}$ \\
\hline Total & $90(29,3)$ & & & $32(10,4)$ & & \\
\hline Sexo & & & $<0,001$ & & & 0,580 \\
\hline Masculino & $65(33,1)$ & 1 & & $22(33,9)$ & 1 & \\
\hline Feminino & $25(59,5)$ & $1,79(1,30-2,47)$ & & $10(40,0)$ & $1,18(0,65-2,14)$ & \\
\hline Idade (anos) & & & $0,001^{* *}$ & & & $0,007^{* *}$ \\
\hline$\leq 14$ & $9(9,1)$ & 1 & & $3(75)$ & 1 & \\
\hline $15-17$ & $14(18,9)$ & $2,08(0,73-5,93)$ & & $7(50)$ & $0,67(0,31-1,45)$ & \\
\hline $18-21$ & $72(60)$ & $6,6(2,56-16,99)$ & & $22(30,6)$ & $0,41(0,21-0,80)$ & \\
\hline Vínculo com a escola & & & $<0,001$ & & & 0,438 \\
\hline Estava estudando & $11(13,9)$ & 1 & & $5(45,5)$ & 1 & \\
\hline $\begin{array}{l}\text { Nunca estudou/ parou } \\
\text { de estudar }\end{array}$ & $79(49,7)$ & $3,57(2,02-6,32)$ & & $27(34,2)$ & $0,75(0,37-1,54)$ & \\
\hline Morar com a família & & & 0,001 & & & 0,466 \\
\hline Sim & $18(21,4)$ & 1 & & $5(27,8)$ & 1 & \\
\hline Não & $72(46,8)$ & $2,18(1,40-3,40)$ & & $27(37,5)$ & $1,35(0,60-3,03)$ & \\
\hline Anos em situação de rua & & & $<0,001^{* *}$ & & & 0,354 \\
\hline$\leq 4$ & $27(23,9)$ & 1 & & $7(25,9)$ & 1 & \\
\hline 5 a 9 & $34(43,6)$ & $1,82(1,20-2,76)$ & & $12(35,3)$ & $1,36(0,62-2,99)$ & \\
\hline$\geq 10$ & $29(61,7)$ & $2,58(1,73-3,85)$ & & $13(44,8)$ & $1,73(0,81-3,69)$ & \\
\hline Horas na rua por dia & & & $<0,001^{* *}$ & & & 0,167 \\
\hline$\leq 7$ & $11(16,7)$ & 1 & & $3(27,3)$ & 1 & \\
\hline $8-14$ & $19(35,2)$ & $2,11(1,10-4,04)$ & & $10(52,6)$ & $1,93(0,67-5,58)$ & \\
\hline$\geq 15$ & $60(50,9)$ & $3,05(1,73-5,38)$ & & $19(31,7)$ & $1,16(0,41-3,28)$ & \\
\hline $\begin{array}{l}\text { Idade } 1^{\text {a }} \text { relação sexual } \\
\text { (anos) }\end{array}$ & & & 0,920 & & & 0,265 \\
\hline$\leq 11$ & $19(38,8)$ & 1 & & $8(42,1)$ & 1 & \\
\hline $12-14$ & $45(37,2)$ & $0,96(0,63-1,46)$ & & $17(37,8)$ & $0,90(0,47-1,72)$ & \\
\hline $15-17$ & $23(37,7)$ & $0,97(0,60-1,57)$ & & $5(21,7)$ & $0,52(0,20-1,33)$ & \\
\hline $18-21$ & $3(50)$ & $1,29(0,54-3,10)$ & & $2(66,7)$ & $1,59(0,60-4,15)$ & \\
\hline $\begin{array}{l}\text { Número de parceiros no } \\
\text { último ano }\end{array}$ & & & 0,009 & & & 0,675 \\
\hline$\leq 10$ & $67(34,4)$ & 1 & & $23(34,3)$ & 1 & \\
\hline$>10$ & $23(56,1)$ & $1,63(1,17-2,28)$ & & $9(39,1)$ & $1,14(0,62-2,10)$ & \\
\hline $\begin{array}{l}\text { Teve parceiro/a fixo/a no } \\
\text { último ano }\end{array}$ & & & 0,019 & & & 0,895 \\
\hline Não & $19(26,0)$ & 1 & & $7(36,8)$ & 1 & \\
\hline Sim & $71(43,29)$ & $1,66(1,09-2,55)$ & & $25(35,2)$ & $0,96(0,39-1,87)$ & \\
\hline $\begin{array}{l}\text { Carregou preservativo } \\
\text { consigo no último mês }\end{array}$ & & & 0,885 & & & 0,992 \\
\hline Não & $31(37,4)$ & 1 & & $11(35,5)$ & 1 & \\
\hline $\operatorname{Sim}$ & $59(38,3)$ & $1,03(0,73-1,45)$ & & $21(35,6)$ & $1,00(0,56-1,81)$ & \\
\hline $\begin{array}{l}\text { Fez sexo em troca de } \\
\text { dinheiro ou favores }\end{array}$ & & & $<0,001$ & & & 0,679 \\
\hline Não & $56(31,1)$ & 1 & & $19(33,9)$ & 1 & \\
\hline $\operatorname{Sim}$ & $34(58,6)$ & $1,88(1,39-2,56)$ & & $13(38,2)$ & $1,13(0,64-1,98)$ & \\
\hline Número de gravidezes & & & - & & & $<0,001$ \\
\hline 1 & - & - & & $10(19,2)$ & 1 & \\
\hline$\geq 2$ & - & - & & $22(57,9)$ & $3,01(1,61-5,61)$ & \\
\hline
\end{tabular}


Tabela 2. continuação

\begin{tabular}{|c|c|c|c|c|c|c|}
\hline \multirow{2}{*}{ Variável } & \multicolumn{3}{|c|}{ Experiência de Gravidez } & \multicolumn{3}{|c|}{ Experiência de Aborto } \\
\hline & $\mathrm{N}(\%)$ & RP (IC95\%) & $p^{*}$ & $\mathrm{~N}(\%)$ & RP (IC95\%) & $p^{*}$ \\
\hline $\begin{array}{l}\text { Idade na primeira } \\
\text { gravidez (anos) }\end{array}$ & & & - & & & $0,005^{* *}$ \\
\hline$\leq 14$ & - & - & & $14(58,3)$ & $2,92(1,24-6,88)$ & \\
\hline $15-17$ & - & - & & $13(31,7)$ & $1,59(0,64-3,93)$ & \\
\hline $18-21$ & - & - & & $5(20)$ & 1 & \\
\hline
\end{tabular}

$N$ : frequência absoluta do desfecho nesta categoria; \%: prevalência do desfecho nesta categoria; RP: razão de prevalências; IC95\%: intervalo de confiança de $95 \%$. * Teste de Wald para heterogeneidade; ${ }^{* *}$ Teste de Wald para tendência linear.

Tabela 3. Fatores independentemente associados à experiência de gravidez entre crianças, adolescentes e jovens em situação de rua, nas cidades de Porto Alegre e Rio Grande/RS - Análise ajustada.

\begin{tabular}{|c|c|c|}
\hline Variável & RP (IC95\%) & $p$ \\
\hline Sexo & & $<0,001$ \\
\hline Masculino & 1 & \\
\hline Feminino & $1,70(1,30-2,22)$ & \\
\hline Idade (anos) & & $<0,001^{* *}$ \\
\hline$\leq 14$ & 1 & \\
\hline $15-17$ & $1,81(0,63-5,16)$ & \\
\hline $18-21$ & $6,04(2,35-15,52)$ & \\
\hline Vínculo com a escola & & $0,05^{*}$ \\
\hline Estava estudando & 1 & \\
\hline Nunca estudou/parou de estudar & $1,83(0,99-3,38)$ & \\
\hline Anos em situação de rua & & $0,006^{* *}$ \\
\hline$\leq 4$ & 1 & \\
\hline 5 a 9 & $1,52(1,05-2,20)$ & \\
\hline$\geq 10$ & $1,61(1,12-2,29)$ & \\
\hline Número de parceiros sexuais no último ano & & 0,035 \\
\hline$\leq 10$ & 1 & \\
\hline$>10$ & $1,41(1,02-1,94)$ & \\
\hline Teve parceiro fixo no último ano & & 0,008 \\
\hline Não & 1 & \\
\hline Sim & $1,66(1,13-2,42)$ & \\
\hline
\end{tabular}

$\mathrm{Na}$ análise ajustada para o aborto (Tabela 4), ter duas ou mais experiências de gravidez e não morar com a família mostraram-se como fatores de risco (RP: 3,70 IC95\%: 1,87-7,30; RP: 2,26 IC95\%: 1,10-4,63, respectivamente). O aumento da idade do indivíduo apareceu como um fator de proteção ( $p$ de tendência linear $=0,007$ ). A variável "idade da primeira gravidez" perdeu o efeito encontrado na análise bivariada. Apesar de manter-se no modelo da regressão para controle de confusão, a variável "anos em situação de rua" não apresentou diferença estatisticamente significativa.

\section{Discussão}

A prevalência de experiência de gravidez nas crianças, adolescentes e jovens em situação de rua foi elevada (29,3\%), se comparada a estudos com adolescentes em desenvolvimento típico dessa faixa etária ${ }^{1-3,13}$. Em função da própria etapa de desenvolvimento e da situação de vulnerabilidade inerente ao contexto de rua, adolescentes e jovens nesta situação estão expostos a uma série de riscos sociais e ambientais ${ }^{1}$.

Quando analisado por sexo, a alta prevalência de experiência de gravidez encontrada no pre- 
Tabela 4. Fatores independentemente associados à experiência de aborto entre crianças, adolescentes e jovens em situação de rua, nas cidades de Porto Alegre e Rio Grande (RS) - Análise ajustada.

\begin{tabular}{lcc}
\hline \multicolumn{1}{c}{ Variável } & $\mathbf{R P}(\mathbf{I C 9 5} \%)$ & $\boldsymbol{p}$ \\
\hline Idade (anos) & 1 & $0,007^{\star}$ \\
$\leq 14$ & $0,67(0,31-1,45)$ & \\
$15-17$ & $0,41(0,21-0,79)$ & \\
$18-21$ & & 0,026 \\
Morar com a família & 1 & \\
$\quad$ Sim & $2,26(1,10-4,63)$ & \\
Não & 1 & $0,05^{*}$ \\
Anos em situação de rua & \\
$\leq 4$ & $1,45(0,67-3,11)$ & \\
5 a 9 & $2,41(0,99-5,83)$ & \\
$\geq 10$ & \multicolumn{2}{c}{$<0,001$} \\
Número de gravidezes & 1 & \\
1 & $3,70(1,87-7,30)$ & \\
$\geq 2$ &
\end{tabular}

RP: razão de prevalências; IC95\%: intervalo de confiança de 95\%. Regressão de Poisson com ajuste robusto da variância, com as variáveis que se mantiveram no modelo final $(\mathrm{p} \leq 0,2)$. * Wald test para tendência linear.

sente estudo $(59,5 \%$ entre meninas e $33,1 \%$ entre meninos) foi superior ao encontrado em estudos prévios realizados na Ucrânia ( $41,7 \%$ para as meninas e $23,5 \%$ para os meninos com idades entre 15 e 24 anos $^{10}$ ) e no Brasil, que registrou prevalência de $51,9 \%$ entre as meninas e $23,2 \%$ entre os meninos ${ }^{14}$. Apesar da literatura apontar que meninas têm maior quantidade de experiências de gravidez do que os meninos ${ }^{10,14}$, isso pode ser devido à natureza do fenômeno. Nos indivíduos do sexo feminino, esta experiência é sentida diretamente, em função de uma série de mudanças corporais e fisiológicas do processo de gestação. No caso dos meninos, ocorre de forma indireta: ele é informado - ou não - por uma terceira pessoa sobre a gravidez. Por isso, é possível que este fenômeno possa ter ocorrido diversas vezes na vida dos meninos, mas nunca ter sido informado, subestimando a real prevalência para os participantes do sexo masculino.

A população deste estudo é predominantemente do sexo masculino, com vínculos frágeis ou inexistentes tanto com a escola como com a família ${ }^{17}$. Considerando que a população-alvo é constituída por crianças, adolescentes e jovens de até 21 anos de idade, o fato de quase metade da amostra estar há 5 anos ou mais em situação de rua ressalta a vulnerabilidade desse grupo. Isto fica evidente nos dados sobre início precoce da vida sexual deste estudo, sexo em troca de dinheiro, experiência de gravidez, idade da primeira gravidez e aborto, em níveis superiores àqueles encontrados na população de adolescentes em desenvolvimento típico ${ }^{31}$.

Houve um aumento na probabilidade de ter a experiência de gravidez conforme aumento na idade. Isso pode ser explicado por um maior tempo de exposição a todos os fatores de vulnerabilidade envolvidos. Da mesma forma, quanto mais anos em situação de rua, maior a probabilidade de ter experienciado a gravidez. Este resultado pode ser explicado pelo modelo de amplificação de risco: Quanto mais cedo o jovem romper com o sistema familiar, mais amplificado o risco de desenvolver uma série de desfechos negativos para a saúde, como a gravidez indesejada ${ }^{6}$.

Os resultados também indicaram associação entre o número de parceiros sexuais no último ano e experiência de gravidez, possivelmente explicada pela tendência de não uso de preservativos $^{8}$. Além disso, houve um aumento na probabilidade de ter experiência de gravidez entre aqueles que tiveram parceiro fixo no último ano. Este resultado pode ser devido à uma crença disseminada na população brasileira, de que o não uso de preservativos seria uma prova de fidelidade $^{32,33}$. Em se adotando o preservativo como principal método contraceptivo desta população, a falta deste aumenta a chance de gravidez ${ }^{34}$. Ao contrário do esperado, morar ou não com a família, assim como o vínculo com a escola não estiveram independentemente associados (análise ajustada) ao desfecho gravidez, contrariando os achados de outros estudos ${ }^{16-17}$, ainda que encontraram associação nas análises bivariadas. Quase todos estudos identificados que apontam associações independentes não apresentam análise estatística controlando para possíveis variáveis confundidoras. Isto sugere que, possivelmente, na presente amostra, vinculação familiar e vinculação escolar estejam associadas com alguma das variáveis que se mantiveram no modelo.

Pesquisas sobre fatores associados ao aborto na população em situação de rua são escassas. No Brasil, foi identificada uma pesquisa com meninos e meninas em situação de rua, de 9 a 18 anos, a qual encontrou que $26,4 \%$ das meninas já havia abortado $^{14}$. Tal valor foi superior ao observado no presente estudo, que registrou o mesmo desfecho em 17,2\% do total de meninas entrevistadas. Pode-se pensar nesta redução da prevalência de aborto como estando associada a políticas de saúde implementadas através do Sistema Único 
de Saúde, as quais aumentaram o acesso a métodos contraceptivos e ao atendimento médico especializado para esta população. Outra possibilidade é que esta diferença seja devido a divergências metodológicas, que limitam a comparação. De qualquer forma, esta prevalência permanece muito elevada, levando em consideração que $2,5 \%$ das mulheres em desenvolvimento típico tinham abortado com idades entre 12 e 17 anos $^{15}$. Somado a isso, dentre os adolescentes e jovens que já abortaram, $43,7 \%$ provocaram o aborto na primeira vez em que ocorreu. No país, o aborto não constitui crime em apenas duas situações, quando a gravidez oferecer risco de vida à gestante ou quando resultar de estupro e a gestante consentir a intervenção. Todas as outras formas de aborto são caracterizadas como crime. Portanto, relatar alguma prática de aborto que não esteja prevista em lei é declarar o cometimento de um crime, fato que pode ter interferido no autorrelato dos participantes, e ter subestimado a real prevalência.

Ter relatado duas ou mais experiências de gravidez esteve associado a um aumento na probabilidade de ter experienciado o aborto. É possível que uma parcela expressiva dos casos de gravidez possam ser gestações indesejadas. Esse resultado é um reflexo do reduzido conhecimento sobre contracepção inerente a essa população ${ }^{14}$, bem como da dificuldade de acesso a diferentes métodos contraceptivos, o que torna o aborto um dos principais métodos para lidar com a gestação indesejada. Além disso, quanto mais velho o entrevistado, menor a chance de aborto. Isso também ocorre na população geral ${ }^{15}$. Ao envelhecer, adolescentes podem manifestar o desejo de engravidar ${ }^{35}$, não querendo interromper a gestação, o que pode se tornar a constituição de uma nova família. Após o ajuste para confusão, aqueles que não moravam com a família também tiveram uma probabilidade maior de ter experienciado o aborto. Este resultado pode estar relacionado com a sensação e efetivo apoio na condução da gestação e criação do bebê por parte da família ${ }^{6}$.

Por ser uma pesquisa com o delineamento transversal não é possível estabelecer relação temporal, não permitindo inferência de causalidade, apenas podendo indicar uma associação entre as variáveis independentes e o desfecho. Os resultados estão, portanto, sujeitos ao viés de causalidade reversa. Outra limitação é o viés de recordatória, uma vez que este estudo fez perguntas sobre o passado desses indivíduos, o que pode ter levado a algum esquecimento. Da mesma forma, apesar dos cuidados metodológicos e éticos, o viés de não-aceitação (ou falsa resposta) pode ter ocorrido, pois como foram entrevistas diretas sobre comportamentos estigmatizados e ilegais, alguns participantes podem ter se resguardado em responder algumas perguntas. Entretanto, estes dois últimos vieses provavelmente operaram subestimando as estimativas. Se houve um prejuízo para o estudo foi o de não encontrar possíveis associações. Por fim, mesmo apontando o delineamento utilizado como uma limitação, a realização do estudo transversal com esses jovens pode ser vantajosa e a melhor opção, em função de ser uma população itinerante e mutável.

Diferenças culturais e metodológicas podem estar presentes nos estudos com crianças, adolescentes e jovens em situação de rua. Pesquisas com a população em situação de rua apresentam variabilidade na definição operacional, diferentes faixas etárias e técnicas de amostragem, o que pode dificultar a comparabilidade. Em função disso, para a presente pesquisa se teve o cuidado de empregar critérios para definir população em situação de rua definidos e utilizados anteriormente em estudos nacionais ${ }^{17,18}$.

\section{Conclusão}

A presente pesquisa respondeu aos objetivos propostos e trouxe contribuições importantes para o conhecimento acerca da saúde sexual e reprodutiva dessa população. A população de crianças, adolescentes e jovens em situação de rua apresenta alta prevalência de experiência de gravidez e de aborto, comparada a pessoas da mesma faixa etária em desenvolvimento típico. Ser do sexo feminino, ter mais de 10 parceiros sexuais no último ano, ter parceiro fixo no último ano $\mathrm{e}$ ter mais idade são fatores que apontam para um aumento na probabilidade de experiência de gravidez nessa população. A prevalência de aborto também foi alta. Esteve mais presente nos participantes mais novos, que não moravam com a família e que tiveram duas ou mais experiências de gravidez. Ficou evidente a necessidade de padronização na maneira de estudar esta população, tanto na definição operacional de situação de rua, critérios de inclusão/exclusão, bem como nas faixas etárias estudadas. Por fim, enfatiza-se a necessidade de se desenvolverem políticas públicas de saúde sexual e reprodutiva específicas para esta população, a fim de protegê-las dos fatores de risco apontados neste estudo, reduzindo os níveis de gravidez indesejada e de abortos, bem como o impacto subsequente sobre o desenvol- 
vimento das crianças, adolescentes e jovens em situação de rua.

Um diferencial metodológico desse estudo foi o uso da técnica de amostragem Respondent-Driven Sampling no grupo de crianças, adolescentes e jovens em situação de rua, com a finalidade de melhor acessar essa população. Não foram identificados outros estudos brasileiros junto a esta população que tenham utilizado esta técnica amostral, sugerindo que este seja o primeiro. Avalia-se que o método RDS mostrou-se bastante efetivo junto à referida população, consideran-

\section{Colaboradores}

L Neiva-Silva trabalhou como coordenador geral da pesquisa. FT Carvalho foi coordenadora assistente. SS Paludo foi coordenadora do projeto na cidade de Rio Grande. Os três trabalharam na elaboração e na implementação do estudo que deu origem ao presente artigo, na coleta, na tabulação e na análise dos dados, na orientação, na redação e na revisão final do artigo. LM Demenech, LR Moreira e AT Oliveira contribuíram no planejamento do artigo, na revisão de literatura, na análise de dados e na redação do artigo. do que foi possível recrutar participantes de áreas até então não acessadas em pesquisas anteriores, devido ao risco dos contextos onde estes sujeitos permaneciam. O método mostrou-se adequado também pela diversidade dos participantes no que diz respeito aos distintos níveis de risco aos quais estavam expostos, bem como sobre o quanto os participantes relataram sentir-se à vontade no processo de estabelecimento de vínculo com os pesquisadores. Recomenda-se que novas pesquisas sejam realizadas com o uso do RDS.

\section{Referências}

1. Boivin JF, Roy E, Haley N, Galbaud du Fort G. The health of street youth: a Canadian perspective. Can J Public Health 2005; 96(6):432-437.

2. Woan J, Lin J, Auerswald C. The health status of street children and youth in low- and middle-income countries: a systematic review of the literature. J Adolesc Health 2013; 53(3):314-321.e12.

3. Greene JM, Ringwalt CL. Pregnancy among three national samples of runaway and homeless youth. J Adolesc Health 1998; 23(6):370-377.

4. Carvalho FT, Neiva-Silva L, Ramos MC, Evans J, Koller SH, Piccinini CA, Page-Shafer K. Sexual and Drug Use Risk Behaviors among Children and Youth in Street Circumstances in Porto Alegre, Brazil. AIDS Behav 2006; 10(4 Supl.):S57-S66.

5. Scappaticci ALSS, Blay SL. Mães adolescentes em situação de rua: uma revisão sistemática da literatura. Revista de psiquiatria do Rio Grande do Sul 2010; 32(1):3-15.

6. Thrane LE, Chen X. Impact of running away on girls pregnancy. J Adolesc 2012; 35(2):443-449.

7. Fundo de População das Nações Unidas (UNFPA). Maternidade precoce: enfrentando o desafio da gravidez na adolescência. Nova York: UNFPA; 2013.

8. Halcón LL, Lifson AR. Prevalence and predictors of sexual risks among homeless youth. Journal of Youth and Adolescence 2004; 33(1):77-80.

9. Haley N, Roy E, Leclerc P, Boudreau JF, Boivin JF. Characteristics of adolescent street youth with a history of pregnancy. J Pediatr Adolesc Gynecol 2004; 17(5):313320.

10. Zapata LB, Kissin DM, Robbins CL, Finnerty E, Skipalska H, Yorick RV, Jamieson DJ, Marchbanks PA, Hillis SD. Multi-city assessment of lifetime pregnancy involvement among street youth, Ukraine. J Urban Health 2011; 88(4):779-792.

11. World Health Organization (WHO). Unsafe abortion: Global and regional estimates of the incidence of unsafe abortion and associated mortality in 2003. Geneva: WHO; 2007. 
12. Brasil. Instituto Brasileiro de Geografia e Estatística (IBGE). Censo Demográfico 2010. [acessado 2015 Jun 8]. Disponível em: http://www.censo2010.ibge.gov.br.

13. Schiro EDB, Dias ACG, Neiva-Silva L, Nieto CJ, Koller SH. Características familiares y apoio percebido entre adolescentes brasileños com y sin experiência de embarazo. Avances en Psicología Latinoamericana 2012; 30(1):66-82.

14. Raffaelli M, Campos R, Merritt AP, Siqueira E, Antunes CM, Parker R, Greco M, Greco D, Halsey N. Sexual practices and attitudes of street youth in Belo Horizonte, Brazil. Street Youth Study Group. Soc Sci Med 1993; 37(5):661-670.

15. Diniz D, Medeiros M. Aborto no Brasil: uma pesquisa domiciliar com técnica de urna. Cien Saude Colet 2010; 15(Supl. 1):959-966.

16. Thompson SJ, Bender KA, Lewis CM, Watkins R. Runaway and pregnant: risk factors associated with pregnancy in a national sample of runaway/homeless female adolescents. J Adolesc Health 2008; 43(2):125-132.

17. Noto AR, Galduróz JCF, Nappo SA, Fonseca, AM, Carlini CMA, Moura YG, Carlini EA, organizadores. Levantamento nacional sobre o uso de drogas entre crianças e adolescentes em situação de rua nas 27 capitais brasileiras, 2003. São Paulo: Centro Brasileiro de Informações sobre Drogas Psicotrópicas; 2004.

18. Neiva-Silva L, Koller SH. A rua como contexto de desenvolvimento. In: Lordelo ER, Carvalho AMA, Koller $\mathrm{SH}$, organizadores. Infância brasileira e contextos de desenvolvimento. São Paulo, Salvador: Casa do Psicólogo, Universidade Federal da Bahia; 2002. p. 205-230.

19. Brasil. Lei no 8.069 de 13 de julho de 1990. Dispõe sobre o Estatuto da Criança e do Adolescente e dá outras providências. Diário Oficial da União 1990; 16 jul.

20. Neiva-Silva L. Uso de Drogas entre crianças e adolescentes em situação: um estudo longitudinal [tese] Porto Alegre: Universidade Federal do Rio Grande do Sul; 2008.

21. Neiva-Silva L, Carvalho FT, Paludo S, Koller SH, Ramos MC, Portolan KCC. Estudo comportamental com crianças e adolescentes em situação de rua em Porto Alegre e Rio Grande: Uso da técnica de Respondent Driven Sampling (RDS) para a identificação de comportamentos sexuais de risco e uso de drogas [Relatório de pesquisa]. Brasília: Ministério da Saúde; 2010.

22. Heckathorn DD. Respondent-Driven Samplimg: a new approach to the study of hidden populations. Social problems 1997; 44(2):174-199.

23. Johnston LG, Thurman TR, Mock N, Nano L, Carccani V. Respondent-Driven Samplimg: a new method for studying street children with findings from Albania. Vunerable Children and Youth Studies 2010; 5(1):1-11.

24. Decker MR, Marshall BD, Emerson M, Kalamar A, Covarrubias L, Astone N, Wang Z, Gao E, Mashimbye L, Delany-Moretlwe S, Acharya R, Olumide A, Ojengbede O, Blum RW, Sonenstein FL. Respondent-driven sampling for an adolescent health study in vulnerable urban settings: a multi-country study. J Adolesc Health 2014; 55(6 Supl.):S6-S12.
25. Heckathorn DD. Respondent-Driven Sampling II: Deriving Valid Population Estimates from Chain-Referral Samples of Hidden Populations. Social Problems 2002; 49(1):11-34.

26. Respondent-Driven Sampling Analysis Tool (RDSAT) [computer program]. Version 7.1. Ithaca: Cornell University; 2012.

27. Salganick MJ, Heckathorn DD. Sampling and Estimation in Hidden Populations Using Respondent-Driven Sampling. Sociological Methodology 2004; 34:193-239.

28. IBM SPSS Statistics for Windows [computer program]. Version 17.0. Armonk: IBM Corp; 2008.

29. Stata Statistical Software: Release 13 [computer program]. College Station: StataCorp LP; 2013.

30. Barros AJ, Hirakata VN. Alternatives for logistic regression in cross-sectional studies: an empirical comparison of models that directly estimate the prevalence ratio. BMC Med Res Methodol 2003; 3:21.

31. Oliveira-Campos M, Nunes ML, Madeira FC, Santos MG, Bregmann SR, Malta DC, Giatti L, Barreto SM. Comportamento sexual em adolescentes brasileiros, Pesquisa Nacional de Saúde do Escolar (PeNSE 2012). Rev. Bras. Epidemio. 2014; (Supl. PeNSE):116-130.

32. Almeida MCC, Aquino EML, Gaffikin L, Magnani RJ. Uso de contracepção por adolescentes de escolas públicas na Bahia. Rev Saude Publica 2003; 37(5):566-575.

33. Geluda K, Bosi MLM, Cunha AJLA, Trajman A. "Quando um não quer, dois não brigam”: um estudo sobre o não uso constante de preservativo masculino por adolescentes do Município do Rio de Janeiro, Brasil. Cad Saude Publica 2006; 22(8):1671-1680.

34. Winetrobe H, Rhoades H, Barman-Adhikari A, Cederbaum J, Rice E, Milburn N. Pregnancy attitudes, contraceptive service utilization, and other factors associated with Los Angeles homeless youths' use of effective contraception and withdrawal. J Pediatr Adolesc Gynecol 2013; 26(6):314-322.

35. Sipsma HL, Ickovics JR, Lewis JB, Ethier KA, Kershaw TS. Adolescent pregnancy desire and pregnancy incidence. Womens Health Issues 2011; 21(2):110-116.

Artigo apresentado em 30/12/2015

Aprovado em 22/06/2016

Versão final apresentada em 24/06/2016 